Supporting Information for

\title{
A Study of $\mathrm{Ba}_{3} \mathrm{M}^{\mathrm{II}} \mathbf{M}^{\mathrm{IV}} \mathrm{WO}_{9}\left(\mathrm{M}^{\mathrm{II}}=\mathrm{Ca}, \mathrm{Zn} ; \mathrm{M}^{\mathrm{IV}}=\mathrm{Ti}, \mathrm{Zr}\right)$ Perovskite Oxides: Competition between $3 \mathrm{C}$ and $6 \mathrm{H}$ Perovskite Structures
}

Rohini Mani, P. Selvamani, Joby E. Joy, J. Gopalakrishnan*, Tapas Kumar Mandal

*To whom correspondence should be addressed.

E-mail : gopal@sscu.iisc.ernet.in. 
Table S1

Powder XRD data for $3 \mathrm{C} \mathrm{Ba}_{3} \mathrm{ZnTiWO}_{9}$

\begin{tabular}{ccccccc}
\hline $\mathrm{h}$ & $\mathrm{k}$ & $\mathrm{l}$ & $\mathrm{d}_{\mathrm{obs}}(\AA)$ & $\mathrm{d}_{\mathrm{calc}}{ }^{a}(\AA)$ & $\mathrm{I}_{\mathrm{obs}}$ & $\mathrm{I}_{\mathrm{calc}}^{b}$ \\
\hline-1 & 0 & 1 & 4.666 & 4.679 & 8 & 6 \\
-1 & 1 & 0 & 4.009 & 4.017 & 3 & 2 \\
2 & 0 & 0 & 2.862 & 2.856 & 100 & 100 \\
-1 & 0 & 3 & 2.442 & 2.445 & 5 & 3 \\
-2 & 0 & 2 & 2.338 & 2.340 & 4 & 4 \\
0 & 2 & 2 & 2.319 & 2.317 & 5 & 6 \\
0 & 0 & 4 & 2.026 & 2.024 & 30 & 35 \\
-3 & 0 & 1 & 1.860 & 1.856 & 2 & 2 \\
2 & 2 & 2 & 1.797 & 1.796 & 1 & 1 \\
-2 & 0 & 4 & 1.655 & 1.655 & 23 & 20 \\
3 & 1 & 2 & 1.641 & 1.645 & 8 & 10 \\
-1 & 0 & 5 & 1.561 & 1.560 & 1 & 2 \\
4 & 0 & 0 & 1.434 & 1.428 & 12 & 15 \\
2 & 2 & 4 & 1.422 & 1.423 & 5 & 7 \\
1 & 4 & 0 & 1.371 & 1.371 & 2 & 1 \\
-1 & 1 & 6 & 1.282 & 1.280 & 8 & 10 \\
-4 & 0 & 4 & 1.171 & 1.170 & 3 & 4 \\
0 & 5 & 2 & 1.085 & 1.088 & 8 & 5 \\
& & & & & & \\
\hline
\end{tabular}

${ }^{a} \mathrm{a}=5.712(5) ; \mathrm{b}=5.650(3) \mathrm{c}=8.094(4) \AA ; \beta=90.31(6)^{\circ}$.

${ }^{b}$ Calculated by POWDERCELL using the position parameters of $\mathrm{Sr}_{2} \mathrm{CoWO}_{6}$ (monoclinic) (Reference 20). 
Table S2

Powder XRD data for $6 \mathrm{H} \mathrm{Ba}_{3} \mathrm{ZnTiWO}_{9}$

\begin{tabular}{|c|c|c|c|c|c|c|}
\hline $\mathrm{h}$ & $\mathrm{k}$ & 1 & $\mathrm{~d}_{\mathrm{obs}}(\AA)$ & $\mathrm{d}_{\text {calc }}{ }^{a}(\AA)$ & $\mathrm{I}_{\mathrm{obs}}$ & $\mathrm{I}_{\text {calc }}^{b}$ \\
\hline 0 & 0 & 2 & 6.99 & 7.03 & 4 & 3 \\
\hline 1 & 0 & 1 & 4.689 & 4.714 & 7 & 5 \\
\hline 1 & 0 & 2 & 4.063 & 4.077 & 4 & 2 \\
\hline 0 & 0 & 4 & 3.515 & 3.517 & 5 & 3 \\
\hline 1 & 0 & 3 & 3.416 & 3.421 & 15 & 10 \\
\hline 1 & 0 & 4 & 2.880 & 2.877 & 100 & 100 \\
\hline 1 & 1 & 2 & 2669 & 2.672 & 1 & 1 \\
\hline 1 & 0 & 5 & 2.455 & 2.452 & 11 & 7 \\
\hline 2 & 0 & 2 & 2.355 & 2.357 & 7 & 3 \\
\hline 1 & 1 & 4 & 2.234 & 2.232 & 5 & 4 \\
\hline 2 & 0 & 3 & 2.208 & 2.207 & 11 & 5 \\
\hline 2 & 0 & 4 & 2.040 & 2.039 & 24 & 23 \\
\hline 2 & 1 & 1 & 1.871 & 1.874 & 11 & 10 \\
\hline 2 & 1 & 2 & 1.827 & 1.826 & 1 & 1 \\
\hline 0 & 0 & 8 & 1.756 & 1.758 & 3 & 3 \\
\hline 3 & 0 & 0 & 1.669 & 1.668 & 22 & 26 \\
\hline 3 & 0 & 3 & 1.572 & 1.571 & 7 & 9 \\
\hline 3 & 0 & 4 & 1.508 & 1.507 & 4 & 5 \\
\hline 1 & 1 & 8 & 1.498 & 1.502 & 4 & 2 \\
\hline 2 & 2 & 0 & 1.447 & 1.444 & 9 & 10 \\
\hline 3 & 1 & 1 & 1.382 & 1.381 & 4 & 5 \\
\hline 2 & 2 & 4 & 1.332 & 1.336 & 4 & 2 \\
\hline 3 & 1 & 4 & 1.295 & 1.291 & 5 & 7 \\
\hline 3 & 0 & 8 & 1.211 & 1.210 & 3 & 2 \\
\hline 3 & 2 & 0 & 1.147 & 1.148 & 2 & 1 \\
\hline
\end{tabular}

${ }^{a} \mathrm{a}=5.8030(1) ; \mathrm{c}=14.1696(2) \AA$.

${ }^{b}$ Calculated by POWDERCELL using the position parameters of $\mathrm{Ba}_{3} \mathrm{ZnRu}_{2} \mathrm{O}_{9}$ (Reference 12). 
Table S3

Powder XRD data for $\mathrm{Ba}_{2.36} \mathrm{Sr}_{0.64} \mathrm{ZnTiWO}_{9}$

\begin{tabular}{ccccccc}
\hline $\mathrm{h}$ & $\mathrm{k}$ & $\mathrm{l}$ & $\mathrm{d}_{\mathrm{obs}}(\AA)$ & $\mathrm{d}_{\text {calc }}{ }^{a}(\AA)$ & $\mathrm{I}_{\mathrm{obs}}$ & $\mathrm{I}_{\text {calc }}{ }^{b}$ \\
\hline 1 & 1 & 1 & 4.66 & 4.66 & 11 & 9 \\
2 & 2 & 0 & 2.859 & 2.858 & 100 & 100 \\
3 & 1 & 1 & 2.439 & 2.437 & 4 & 5 \\
2 & 2 & 2 & 2.319 & 2.333 & 4 & 4 \\
4 & 0 & 0 & 2.023 & 2.021 & 27 & 30 \\
3 & 3 & 1 & 1.855 & 1.854 & 2 & 2 \\
4 & 2 & 2 & 1.649 & 1.649 & 31 & 38 \\
4 & 4 & 0 & 1.428 & 1.429 & 13 & 17 \\
5 & 3 & 1 & 1.367 & 1.366 & 1 & 2 \\
6 & 2 & 0 & 1.277 & 1.278 & 11 & 16 \\
& & & & & & \\
\hline
\end{tabular}

${ }^{a} \mathrm{a}=8.082(2) \AA$.

${ }^{b}$ Calculated by POWDERCELL using the position parameters of $\mathrm{Ba}_{2} \mathrm{ZnWO}_{6}$ (Reference 19). 